The Astrophysical Journal, 161:L1-L7, July 1970

(C) 1970. The University of Chicago. All rights reserved. Printed in U.S.A

\title{
DETECTION OF X-RAY EMISSION FROM 3C 273 AND NGC 5128*
}

\author{
C. Stuart Bowyer, Michael Lampton, and John Mack \\ AND \\ Fernando de Mendonca \\ Comissao Nacional de Atividades Espaciais, Brazil \\ Received 1970 June 1
}

Department of Astronomy and Space Sciences Laboratory, University of California, Berkeley

\section{ABSTRACT}

Well-collimated proportional counters sensitive to $1-10-\mathrm{keV} X$-rays have been flown on an attitudecontrolled Aerobee 150 rocket and have detected discrete sources at the positions of the extragalactic objects 3C 273, NGC 5128 (Cen A), and M87. The X-ray emission from Cen A is confined within $2^{\circ}$ of the optical galaxy. The X-ray flux of Cen A falls below the extrapolated radio spectrum.

On 1969 June 14 at $21^{\mathrm{h}} 52^{\mathrm{m}}$ U.T. an Aerobee 150 payload instrumented to detect celestial X-ray sources was launched from Natal, Brazil. An attitude-control system (ACS) was employed to provide a controlled scan of selected regions of the celestial sphere. The data reported here are obtained from a scan over moderate to high galactic latitudes in which the peculiar galaxy NGC 5128 (Cen A), 3C 273, and M87 were observed.

Data were obtained from two proportional counters capable of detecting X-rays with energies in the range from 0.2 to $10 \mathrm{keV}$ and a scintillation counter sensitive to $\mathrm{X}$-rays from 10 to $30 \mathrm{keV}$. As no sources were detected above background in the $10-30-\mathrm{keV}$ range, this part of the instrumentation and the resulting data will not be discussed in detail. The proportional counters used for measurements at lower energies employed continuous gas-flow systems identical in concept to that described in a report of a previous flight (Bowyer, Field, and Mack 1968). Charged-particle background counts were rejected by the use of anticoincident gas counters which covered the backs and sides of the primary detectors. The overall gain of each of the primary counters was held constant by a feedback loop which was capable of varying the system gain over a 10:1 range. The system gain was slaved to a calibration counter with an internal ${ }^{55} \mathrm{Fe}$ source which shared the high voltage and counting gas of the primary detector. In addition to providing continuous control of the gain, the output of the calibration counter was read periodically to monitor the overall stability of the system.

Two different collimators were employed: a hexagonal-honeycomb structure providing a small circular field of view and a stacked-plate structure providing a large fan-shaped field of view. A determination of the field of view of these collimators was made with optical light. Data obtained during the flight on isolated X-ray sources confirmed the optical calibration of the circular collimator and confirmed the calibration of the narrow dimension of the fan-shaped collimator. The window of each detector was nominal, $\frac{1}{8}$-mil Mylar with a conducting Nichrome coating. A laboratory calibration of the transmission of each window was made at 14.6 and $44.6 \AA$. The counting gas was 90 percent argon and 10 percent methane. Relevant parameters for these detectors are provided in Table 1. In-flight verification of these detector parameters was indirectly provided by observations of previously identified X-ray sources. For example, the well-isolated source GX10.7 (Gursky, Gorenstein, and Giacconi 1967) was observed by both detectors midway in this flight. Analyzing our data at $4 \mathrm{keV}$ from this source, we obtain a flux density equal

* Space Sciences Laboratory Series 11, Issue 39. 
to that reported by Gursky et al. (1967) within the statistical errors of the two experiments.

The track of the center of the field of view of each detector is shown in Figure 1. The full width at half-maximum (FWHM) field of view of each detector and the positions of NGC 5128, 3C 273, and M87 are also indicated. Different scan rates were employed over different segments of the track. For $5^{\circ}$ on either side of Cen A (from point $A$ to point $B$ in Fig. 1), a scan rate of $0^{\circ} .518 \mathrm{sec}^{-1}$ was employed. At point $B$, an alignment maneuver was performed which briefly brought Cen $A$ back into the edge of the field of view of the large collimator. A rapid scan was made from point $B$ to point $C$, where another alignment maneuver was carried out. The scan from $5^{\circ}$ before $3 \mathrm{C} 273$ to $5^{\circ}$ beyond $\mathrm{M} 87$ (from point $C$ to point $D$ in Fig. 1) was made at $0^{\circ} .34 \mathrm{sec}^{-1}$. The location of the scan track was obtained from an analysis of the telemetry output of the ACS. Verification of this track to an accuracy of a few degrees was provided by data from an on-board stellar photome-

TABLE 1

DETECTOR PARAMETERS

\begin{tabular}{|c|c|c|c|c|c|}
\hline \multirow[b]{2}{*}{ Collimator } & \multirow{2}{*}{$\begin{array}{l}\text { FIELD OF } \\
\text { VIEW } \\
\text { (FWHM) }\end{array}$} & \multirow{2}{*}{$\begin{array}{c}\text { EFFECTIVE } \\
\text { AREA } \\
\left(\mathrm{cm}^{2}\right)\end{array}$} & \multicolumn{2}{|c|}{$\begin{array}{c}\text { Window Transmission } \\
(\%)\end{array}$} & \multirow{2}{*}{$\begin{array}{c}\text { GAS SURFACE } \\
\text { DENSITY } \\
\left(\mathrm{mg} / \mathrm{cm}^{3}\right)\end{array}$} \\
\hline & & & $14.6 \AA$ & $44 \AA$ & \\
\hline Small. & $1: 6$ (circular) & 332 & 8.8 & 14.4 & Argon, 4.33 \\
\hline Large. . & $3^{\circ} \times 12^{\circ}$ & 347 & 8 & 13.8 & $\begin{array}{l}\text { Argon, } 4.00 \\
\text { Methane, } 0.18\end{array}$ \\
\hline
\end{tabular}

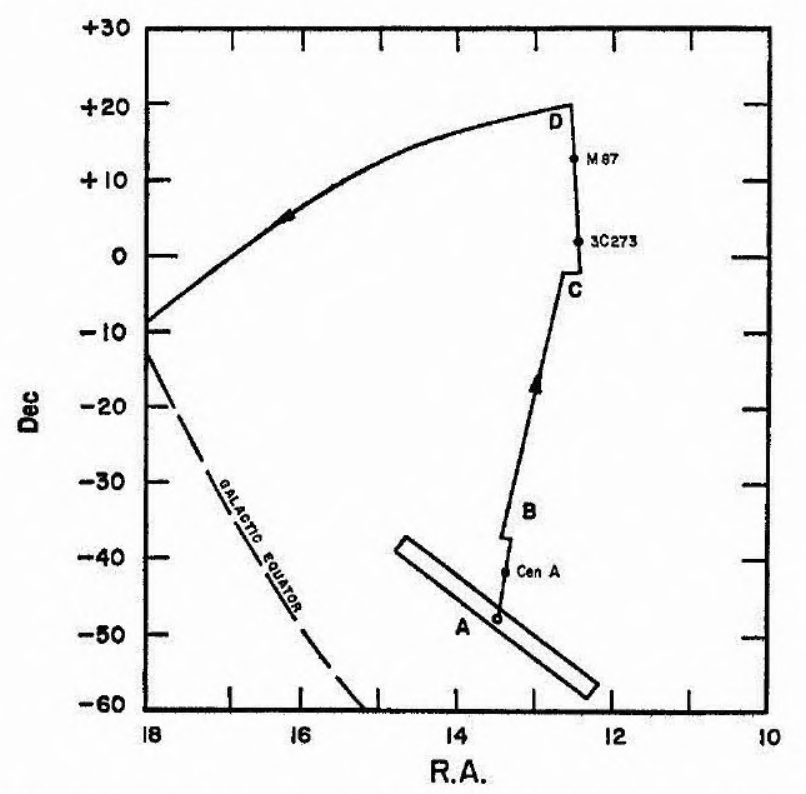

Fic. 1.-Map of the celestial sphere showing the scan path of the center of the field of view of each detector. The size of the field of view of each detector (FWHM) is shown at the beginning of the scan. Solid circles, locations of Cen A, 3C 273, and M87. 
and from magnetometer data. Verification of this track to within a fraction of a jue was provided by the $\mathrm{X}$-ray data, as will be discussed below. Cen $\mathbf{A}$ was observed un altitude of $115 \mathrm{~km}, 3 \mathrm{C} 273$ at $142 \mathrm{~km}$, and M87 at $155 \mathrm{~km}$. The line-of-sight atmeric absorption to these sources as derived from the COSPAR International ReferAtmosphere was 10 percent at $5 \AA$ for Cen A, less than 4 percent at 12 and $44 \AA$ for 273 , and less than 2 percent at these wavelengths for M87.

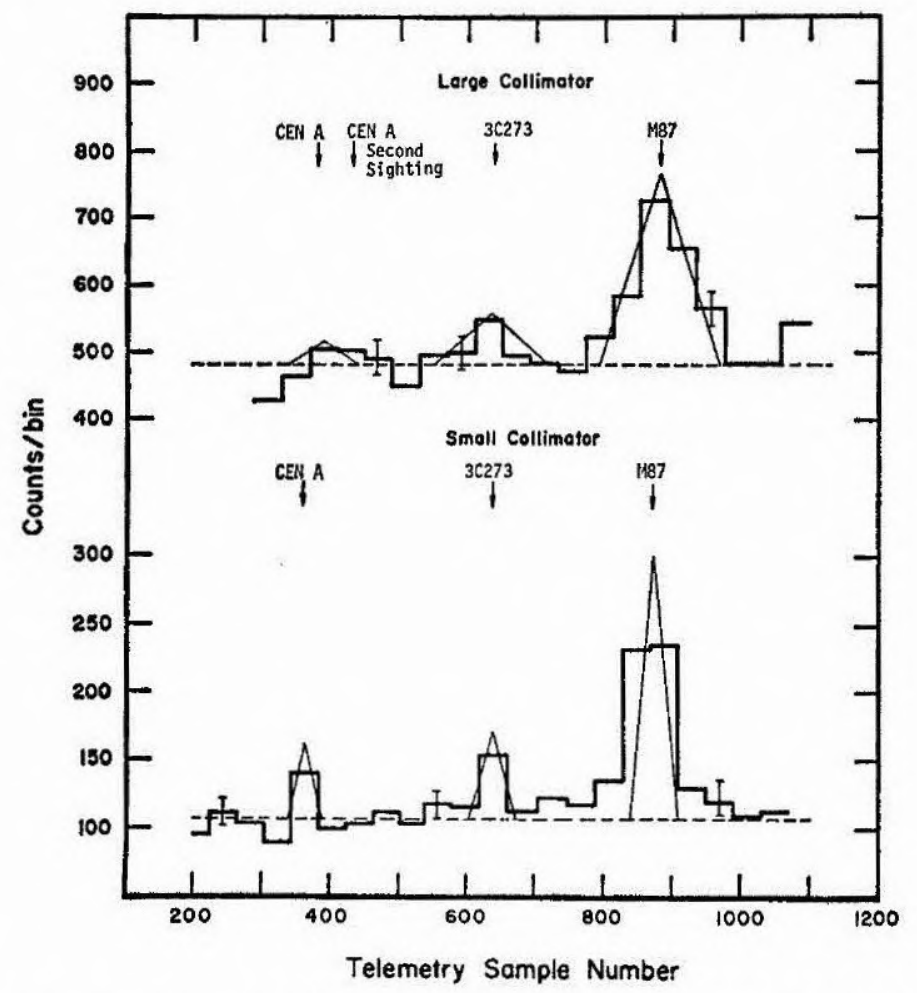

Fic. 2.-X-ray counting rate versus telemetry sample number for the two detectors. Triangular funcions, collimator responses for point sources at the time of the peak X-ray signal. Arrows, times of transit of Cen A and 3C 273 as derived from the ACS data and the location of M87.

Counts from each of the proportional counters were amplified, and pulse heights were analyzed and stored in an on-board memory. The telemetry system provided 7.81 memory readouts per second; for purposes of this analysis, the data from all energy channels were summed and are considered as a single sample. In Figure 2 we have plotted summed blocks of forty data samples as a function of the telemetry sample number. Counting errors of $1 \sigma$ are indicated at several points for each detector.

The positional information derived from the ACS data indicates that the small collimator scanned directly across M87. The peak X-ray intensity from M87 as measured by each detector was the same within the statistical limitations of the data. Because of the extreme difference in collimation between the two detectors in the transverse direction, we can conclude that the location of the scan track of the detector with the circular collimator passed over M87 to within $\pm 0^{\circ} .25$, thus confirming the ACS positional data in this dimension.

A determination of the time of the peak X-ray signals for the three sources was made 
by folding an approximation to the collimator response function through the data. This procedure is quite accurate in the case of M87, where the statistical uncertainties in the data blocks employed were not large. We have indicated the position of M87 based on this analysis by an arrow in Figure 2. A comparison of the time of peak X-ray signal from M87 with the apparent scan position as derived from the ACS data showed a 0.7 roll offset, which falls within the normal range of error of the ACS. We have applied this offset to the remainder of the ACS data and indicate the resulting locations of 3C 273 and Cen $\mathrm{A}$ by arrows in Figure 2. These are shown separately for each detector because the look angles of the two detectors in the roll direction differ slightly.

In Table 2 we list the coordinates of 3C 273 and NGC 5128 along with the location of the peak X-ray signal derived from the data obtained with the tightly collimated detector. The errors in the roll direction were estimated from the relation $\sigma^{2}=\sigma_{r}{ }^{2}+\sigma_{\mathrm{Cen}}{ }^{2}+$ $\sigma_{\mathrm{M} 87^{2}}{ }^{2}+\sigma_{\mathrm{ACs}}^{2}$, where $\sigma_{r}$ is a 1 percent uncertainty in the ACS roll rate measured from the

TABLE 2

COMPARISON OF OPTICAL AND

X-RAY SOURCE POSITION

\begin{tabular}{|c|c|c|}
\hline Object & $\begin{array}{c}\text { Location of } \\
\text { Optical Source }\end{array}$ & $\begin{array}{c}\text { Location of Peak } \\
\text { X-Ray Signal }\end{array}$ \\
\hline $\begin{array}{l}3 \mathrm{C} 273 \ldots \ldots \ldots \\
\text { NGC } 5128 \ldots \ldots\end{array}$ & $\begin{array}{l}a, 12^{\mathrm{b}} 26^{\mathrm{m}} 5 \\
\delta, 2^{\circ} 33 \\
a, 13^{\mathrm{h}} 22^{\mathrm{m}} \cdot 5 \\
\delta,-42^{\circ} .77\end{array}$ & $\begin{array}{l}a, 12^{\mathrm{b}} 26^{\mathrm{m}} \cdot 5 \pm 2^{\mathrm{m}} \cdot 7 \\
\delta, \quad 2^{\circ} .5 \pm 0^{\circ} \cdot 6 \\
a, 13^{\mathrm{h}} 22^{\mathrm{m}} \pm 3^{\mathrm{m}} \\
\delta,-42^{\circ} .8 \pm 0.9\end{array}$ \\
\hline
\end{tabular}

TABLE 3

SignAL CONFIDENCE LEVELS

\begin{tabular}{ccccc}
\hline \hline Object & $\begin{array}{c}\text { Size } \\
\text { Collimator }\end{array}$ & $\begin{array}{c}\text { Signal Excess } \\
\text { over } \\
\text { Background }\end{array}$ & $\begin{array}{c}\text { Probability } \\
\text { of Chance } \\
\text { Occurrence in } \\
15 \text { Samples }\end{array}$ & $\begin{array}{c}\text { Probability of Chance } \\
\text { Occurrences of Both } \\
\text { Signals from a } \\
\text { Particular Source }\end{array}$ \\
\hline 3 C $273 \ldots \ldots$. & Large & $3.1 \sigma$ & $1.5 \times 10^{-2}$ & $4.8 \times 10^{-8}$ \\
Cen A....... & Small & $3.9 \sigma$ & $7.2 \times 10^{-4}$ & $4.8 \times 10^{-8}$ \\
& Large & $1 \sigma$ & 0.993 & $2.2 \times 10^{-4}$ \\
& Small & $3 \sigma$ & $2.1 \times 10^{-2}$ & $2.2 \times 10^{-4}$ \\
\hline
\end{tabular}

location of $\mathrm{M} 87, \sigma_{\mathrm{Cen}}$ and $\sigma_{\mathrm{M} 87}$ are uncertainties in the location of the peak X-ray signal at each source, and $\sigma_{\mathrm{ACS}}$ is the limit cycle range of the ACS in the roll direction. The errors in the pitch direction were taken as $\sigma^{2}=\sigma_{T}{ }^{2}+\sigma_{\mathrm{ACs}}{ }^{2}$, where $\sigma_{T}$ is the possible error in the scan track at M87 as derived above and $\sigma_{\mathrm{ACS}}$ is the limit cycle range of the $\mathrm{ACS}$ in the pitch direction.

The statistical standard deviations of the detected excess fluxes were evaluated by using the relation $\sigma^{2}=S / T_{1}+B / T_{1}+B / T_{2}$, in which the signal $S$ plus background $B$ count rates are observed for a time $T_{1}$ and the background alone is observed for a time $T_{2}$. For the detector with the large collimator, the signal associated with Cen A was taken as only that single block of data observed during the first sighting. The remainder of the data blocks (with the exception of the data clearly associated with M87 and the data block from the direction of $3 \mathrm{C} 273$ ) were used in the assessment of the background. For the small collimator, the Cen A signal and the 3 C 273 signal were taken from the single blocks of data indicated.

In Table 3 we present the signal excess above background along with the probability 
that this signal would have occurred by chance in one of fifteen data blocks. We also indicate the probability that both of these signals would have been observed by chance from a particular direction in the celestial sphere. This probability depends, of course, upon the a priori knowledge of the uniqueness of each of these sources.

The size of the X-ray source at NGC 5128 was evaluated by comparing the X-ray data with the response of an ideal collimator to various diameter sources. The sum $\Sigma_{i}\left(\delta_{i} / \sigma_{i}\right)^{2}$ was calculated for each trial source, where $\delta_{i}$ is the difference between the predicted and the observed signal and $\sigma_{i}$ is the standard deviation of this signal. A point source was found to provide the best fit to the data. Following procedures outlined by Giacconi, Gursky, and van Speybroeck (1968), we found the standard error of the square of the source diameter to be 3.76 square degrees, which indicates that the source is $\leq 1.9$ in diameter.

In Figure 3 we have plotted a portion of the detector scan track on a map of the 31.2$\mathrm{cm}$ radio emission associated with Cen $\mathrm{A}$ (adapted from Cooper, Price, and Cole 1965). Radio brightness contour lines are indicated in degrees Kelvin. The filled circle in the center of the figure indicates the size and position of the optical galaxy. The upper

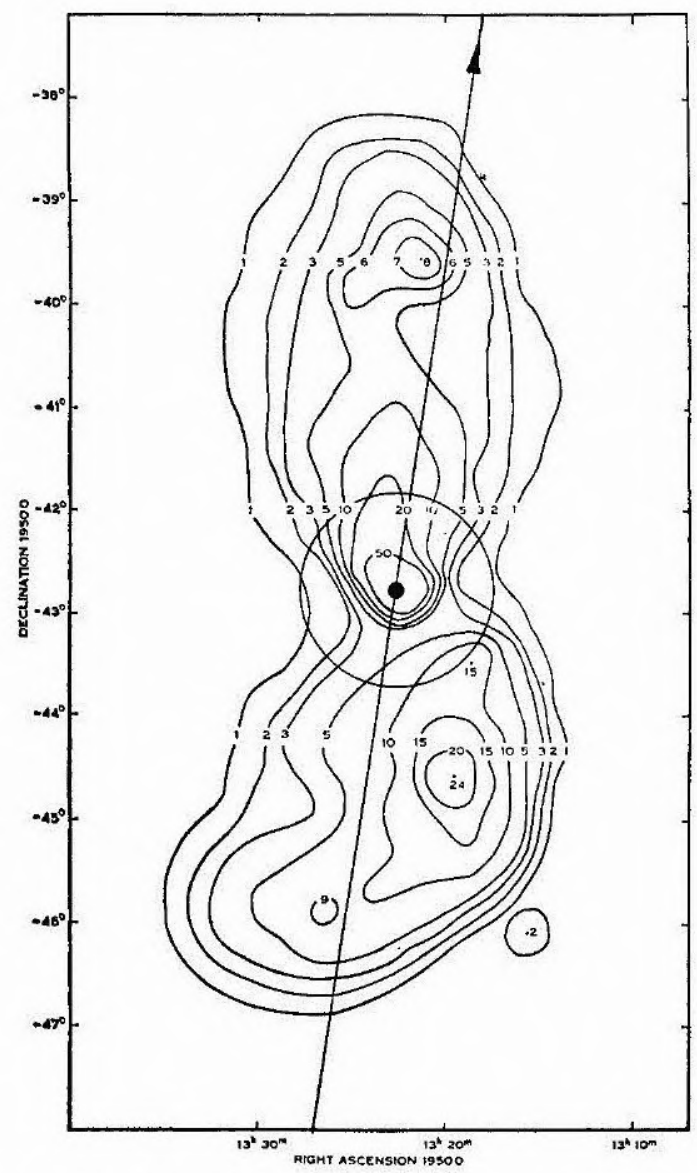

FIG. 3.-Track of the centers of the fields of view of the X-ray detectors plotted on a map of 31.2-cm radio emission from Cen $\mathrm{A}$. The upper limit to the size of the $\mathrm{X}$-ray source is indicated by a circle centered at the most probable source position. 
limit to the size of the X-ray source is shown by a circle centered on the most probable source position. This figure should be considered in connection with the data shown in Figure 2. In that figure, the two blocks of data to each side of the source correspond to $5^{\circ}$ scan segments along the track shown in Figure 3. Since these segments cover the most intense emission regions of the extended radio halo and since no evidence of any signal above background was detected, we conclude that the $\mathrm{X}$-ray emission is localized in the central portion of the object.

The observed count rates of NGC 5128 and 3C 273 in the $\frac{1}{4}-12-\mathrm{keV}$ band were $0.044 \pm$ 0.015 counts $\mathrm{cm}^{-2} \mathrm{sec}^{-1}$ for $3 \mathrm{C} 273$, and $0.039 \pm 0.013$ counts $\mathrm{cm}^{-2} \mathrm{sec}^{-1}$ for Cen $\overline{\mathrm{A}}$. Folding the detector response with an assumed spectral index of -1.0 for each source, we obtain a flux density at $10^{18} \mathrm{~Hz}$ of $1.17 \pm 0.40 \times 10^{-28} \mathrm{erg} \mathrm{cm}^{-2} \mathrm{sec}^{-1} \mathrm{~Hz}^{-1}$ for $3 \mathrm{C}$

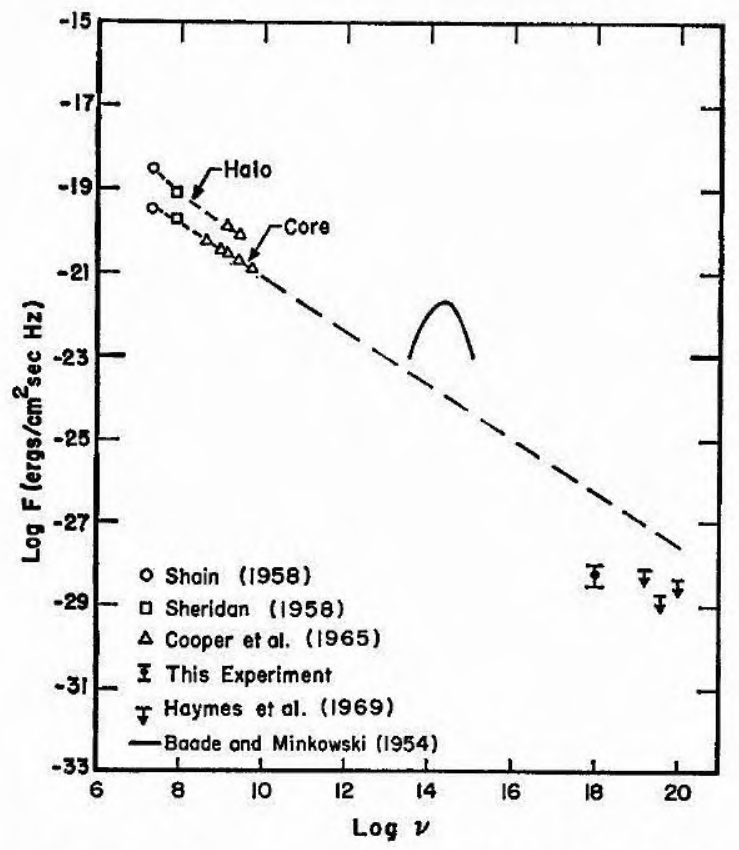

FIG. 4.-Flux density as a function of frequency for NGC 5128. The radio emission (halo and core), optical, and $\mathrm{X}$-ray flux are indicated. Dotted line, extrapolation of the core radio flux to the $\mathrm{X}$-ray region.

273, and $1.04 \pm 0.35 \times 10^{-28} \mathrm{erg} \mathrm{cm}^{-2} \mathrm{sec}^{-1} \mathrm{~Hz}^{-1}$ for Cen $\mathrm{A}$. This corresponds respectively to total energy fluxes of $3 \times 10^{-10}$ and $2.7 \times 10^{-10} \mathrm{erg} \mathrm{cm}^{-2} \mathrm{sec}^{-1}$ in the $1-10 \mathrm{-keV}$ energy range. In Figure 4 we have plotted the frequency distribution of the optical and radio emission from NGC 5128. We have indicated by a dotted line an extrapolation of the core radio flux to $\mathrm{X}$-ray energies. It is clear that the observed $\mathrm{X}$-ray flux falls below this extrapolation. We also indicate upper limits on the $\mathrm{X}$-ray flux obtained from the balloon experiment of Haymes et al. (1969).

In Table 4 we have listed the total X-ray luminosities of $3 \mathrm{C} 273$ and Cen A in the energy band from 1 to $12 \AA$. The distance to 3C 273 was taken as $630 \mathrm{Mpc}$, and the distance to Cen A was taken as $4 \mathrm{Mpc}$, based on $H=75 \mathrm{~km} \mathrm{sec}^{-1} \mathrm{Mpc}^{-1}$ (Sandage 1968). We have also tabulated the visible, infrared, and radio luminosities of these objects where available.

The detection of X-rays from 3C 273 has been previously reported by the NRL group (Friedman and Byram 1967) at about the $1 \sigma$ confidence level (Argyle 1968; Friedman, 
Byram, and Chubb 1968). Firm upper limits to the X-ray intensity of this source have been obtained by an MIT group (Bradt et al. 1967). The flux we observed is less than the upper limit set by the MIT group and is compatible with the NRL measurement within the statistical limitations of the two experiments.

Of the three extragalactic objects from which X-rays were detected in this flight, M87 and $3 C 273$ have jetlike structures at optical wavelengths. While no report has been made of an optical jet in NGC 5128, it is possible that such a feature exists and has been overlooked or is obscured by the dust lane. It is of interest to note that there is an indication of an amorphous extension of NGC 5128 along its axis of rotation (Smith 1970).

It is tempting to conclude that $\mathrm{X}$-ray emission in extragalactic objects may be primarily associated with only the early phases of some violent event occurring in the object. If true, the relatively transient nature of this phenomenon may be due to a rapid

TABLE 4

SOURCE LUMINOSITIES

(ergs per second)

\begin{tabular}{ccccc}
\multicolumn{5}{c}{ (ergs per second) } \\
\hline \hline Object & $\begin{array}{c}\text { X-ray } \\
(1-12 \AA)\end{array}$ & $\begin{array}{c}\text { Visible } \\
(3000-10000 \AA)\end{array}$ & $\begin{array}{c}\text { Infrared } \\
(5-300 \mu)\end{array}$ & $\begin{array}{c}\text { Radio } \\
(0.3 \mathrm{~mm} \text { to } 30 \mathrm{~m})\end{array}$ \\
\hline $3 \mathrm{C} 273 \ldots \ldots$ & $1.4 \times 10^{46}$ & $10^{46}$ & $6.2 \times 10^{48}$ & $2.5 \times 10^{46}$ \\
Cen A $\ldots \ldots$ & $5.0 \times 10^{41}$ & $8 \times 10^{43}$ & $\ldots$ & $1.8 \times 10^{41}$ \\
\hline
\end{tabular}

degradation of the underlying energy source or may be due to a spatial separation of the requisite components of the source mechanism with time.

We wish to thank the staff of the Sounding Rocket Branch of Goddard Space Flight Center for their support. We also wish to thank Dr. Burton Henke for assistance in the calibration of the detector windows. This work was supported by the National Aeronautics and Space Administration under grant NGR 05-003-278 and by the Comissao Nacional de Atividades Espaciais of Brazil.

\section{REFERENCES}

Argyle, E. 1968, Science, 159, 747.

Baade, W., and Minkowski, R. 1954, $A$ p. $J ., 119,215$.

Bowyer, C. S., Field, G. B., and Mack, J. E. 1968, Nature, 217, 32

Bradt, H., Mayer, W., Naranan, S., Rappaport, S., and Spada, G. 1967, A p. J. (Letters), 150, L199.

Cooper, B. F. C., Price, R. M., and Cole, D. J. 1965, Australian J. Phys., 18, 589.

Friedman, H., and Byram, E. T. 1967, Science, 158, 257.

Friedman, H., Byram, E., and Chubb, T. 1968, Science, 159, 748

Giacconi, R., Gursky, H., and van Speybroeck, L. P. 1968, Ann. Rev. Astr. and A p., 6, 373.

Gursky, H., Gorenstein, P., and Giacconi, R. 1967, Ap. J. (Letters), 150, L75.

Haymes, R. C., Ellis, D. V., Fishman, G. J., Glenn, S. W., and Kurfess, J. D. 1969, A p. J. (Letters), 155, L31.

Sandage, A. R. 1968, Ap. J. (Letters), 152, L149.

Shain C. A. 1958, Australian J. Phys., 11, 517.

Sheridan, K. V. 1958, Australian J. Phys., 11, 400.

Smith, M. G. 1970 (private communication). 